\title{
Introduction to the Special Issue on Music and Empathy: Part 2
}

\author{
CAROLINE WADDINGTON \\ University of Hull, $U K$
}

THIS second part of the special issue on music and empathy research invites the reader to explore empathy and musical engagement in various contexts. In the area of music performance, Erin Heisel examines the empathic process employed by singers to embody and portray a character. She proposes a method of role journaling as a means of recording, developing, and reflecting on the process of embodiment. In response to Heisel, Thalia Goldstein considers research from acting and theatre that speaks directly to how embodiment occurs in vocal performance, and suggests that singers may benefit from certain exercises employed by actors in approaching the embodiment of a character. In his commentary, Anton Vishio proposes expanding the kinds of empathy that might be most useful to performers to include cognitive empathy and to engage considerations of musical structure and embodiment. He also proposes expanding Heisel's pedagogical techniques into situations where empathy may be hard to find.

Jonathan Still considers how empathy may, or may not, be implicated in ballet dancers' embodied experiences of meter, and suggests that empathic listening and perspective taking, rather than an appeal to common embodied experiences of meter, may be key to developing better interdisciplinary understanding between musicians and dancers. In response, Arnie Cox reflects on the relationship between embodiment, physical gravity, and the notion of "musical gravity," whilst Robert Hatten considers ways in which meter suggests a virtual environmental field, and argues that the experience of moving up on a downbeat can imply down as gravitational field.

Finally, Elizabeth Haddon and Mark Hutchinson investigate empathy between players in piano duet rehearsal and identify examples of bodily, verbal, emotional and cognitive empathy which facilitate instinctive understanding and tacit communication, unspoken conflict resolution, and the development of creative empathy in relation to the piece of music being rehearsed. In relation to Haddon and Hutchinson's work, Mine Doğanten-Dack emphasizes empathy as both a biologically and culturally embedded phenomenon and as a dynamically emergent experience, and evaluates its epistemic status in explaining human action. Meanwhile, Fred Seddon reflects on the unique role of "written empathy" within the context of empathic relationships and considers how shifts from verbal to non-verbal communication may indicate movement from a "top-down" to "bottom-up" response, and how this shift is related to "empathetic attunement."

I am very grateful to the editors of Empirical Musicology Review, Nicola Dibben and Renee Timmers, for the opportunity to realize this special issue on music and empathy, and to the authors of all target articles and commentaries for their valuable and thought-provoking contributions which made working on the special issue a really enjoyable experience. 